\title{
EEPI: instrumento de evaluación de entornos de proximidad para incluir a las personas sin autonomía en Lima
}

\author{
Félix Cabrera
}

Pontificia Universidad Católica del Perú

cabrera.fi@pucp.edu.pe

\section{Àngel Cebollada}

Universitat Autònoma de Barcelona

angel.cebollada@uab.cat

Recibido: febrero de 2020

Aceptado: diciembre de 2020

Publicado: marzo de 2021

\section{Resumen}

Para diseñar los entornos de proximidad, se utilizan criterios que provienen de la perspectiva de los profesionales y de experimentos en laboratorio, pero no se ha tenido en cuenta realmente las opiniones de la ciudadanía. Tampoco se han analizado simultáneamente a los diferentes tipos de personas ni se han incluido a los grupos con discapacidad visual y cognitiva. Por ello, para evaluar los espacios urbanos, desde una perspectiva holística e inclusiva, se presenta el instrumento EEPI que puede ser empleado en edificaciones, aceras, plazas y cruceros peatonales. Se caracteriza por incorporar a niños, niñas, adultos mayores y personas con discapacidad (motriz, visual y cognitiva); escuchar su voz; provenir de un enfoque mixto; ser útil y fácil de aplicar y transferir, e incluir criterios físicos, personales y sociales. Además, cuenta con dos niveles — planificación y gestión- $\mathrm{y}$, como todo instrumento, es totalmente perfectible y puede dar cabida a otros aspectos.

Palabras clave: movilidad; accesibilidad; espacio público; entorno de proximidad; persona sin autonomía 
Resum. EEPI: instrument d'avaluació d'entorns de proximitat per incloure les persones sense autonomia a Lima

Per dissenyar els entorns de proximitat, s'utilitzen criteris que provenen de la perspectiva dels professionals i d'experiments en laboratori, sense tenir en compte les opinions de la ciutadania. Així mateix, tampoc s'han analitzat simultàniament els diferents tipus de persones, com ara els grups amb discapacitat visual i cognitiva. Per això, per avaluar els espais urbans des d'una perspectiva holística i inclusiva, es presenta l'instrument EEPI, que pot ser emprat en edificacions, voreres, places i passos de vianants. Aquest instrument es caracteritza per incorporar infants, gent gran i persones amb discapacitat (motriu, visual i cognitiva); per escoltar la seva veu; per provenir d'un enfocament mixt; per ser útil i facil d'aplicar i transferir, i per incloure criteris físics, personals i socials. A més, compta amb dos nivells — planificació i gestió- i, com tot instrument, és totalment perfectible i pot donar cabuda a altres aspectes.

Paraules clau: mobilitat; accessibilitat; espai públic; entorn de proximitat; persona sense autonomia

Résumé. EEPI: Instrument pour l'évaluation des environnements de proximité pour l'inclusion des personnes sans autonomie à Lima

Pour concevoir des environnements de proximité, certains critères émergeant du point de vue des professionnels et des expérimentations en laboratoire sont utilisés, mais les avis des citoyens n'ont pas vraiment été pris en compte. Les différents types de personnes n'ont pas non plus été simultanément analysés, et on n'a pas non plus inclus les groupes présentant des déficiences visuelles et cognitives. Pour cette raison, pour évaluer les espaces urbains dans une perspective holistique et inclusive, nous présentons l'instrument EEPI. Il peut être utilisé pour les bâtiments, les trottoirs, les places et les passages pour piétons. Il est caractérisé par l'intégration des garçons, des filles, des personnes âgées et des personnes handicapées (motrices, visuelles et cognitives); il permet d'entendre sa voix; provient d'une approche mixte; il est utile et facile à appliquer ainsi qu'à transférer; et il comprend des critères physiques, personnels et sociaux. Il comporte également deux niveaux : planification et gestion ; et comme tout instrument, il est totalement perfectible et peut intégrer d'autres aspects.

Mots-clés : mobilité; accessibilité; espace public; environnement de proximité ; personne sans autonomie

Abstract. EEPI: Instrument for the evaluation of proximity environments to include people without autonomy in Lima

To design proximity environments, criteria from the perspective of professionals and laboratory experiments are used. However, the opinions of citizens have not really been taken into account, the different types of people have not been analyzed simultaneously, and groups with visual and cognitive disabilities have not been included. For this reason, to evaluate urban spaces from a holistic and inclusive perspective, the EEPI instrument is presented. The EEPI can be used in buildings, sidewalks, squares, and pedestrian crossings. It includes boys, girls, older adults, and people with disabilities (motor, visual, and cognitive) and takes into account their opinions, it is based on a mixed approach, it is useful and easy to apply and transfer, and it also includes physical, personal, and social criteria. The EEPI is also structured on two levels: planning and management. Moreover, like any instrument, it is subject to improvement and can accommodate other aspects.

Keywords: mobility; accessibility; public space; proximity environment; person without autonomy 


\section{Sumario}
1. Introducción
5. Discusión
2. Marco teórico
6. Conclusiones
3. Metodología
Referencias bibliográficas

4. El instrumento EEPI

\section{Introducción}

La evidencia muestra que el desarrollo de la vida urbana y el acceso a la ciudad se asocian directamente a las políticas de movilidad (Herce, 2009) y al diseño y evaluación de los entornos de proximidad (Talavera-García et al., 2014; Gehl, 2014; Fruin, 1971). De acuerdo con el sujeto de estudio, se pueden encontrar diversas propuestas. Por ejemplo, algunas plantean diseñar y evaluar entornos enfocándose solo en el hombre adulto (TRB, 2010), otras en la perspectiva de género (Ortiz Escalante y Gutiérrez Valdivia, 2015) y las más recientes en las necesidades de los grupos sin autonomía, como las personas con discapacidad (Winance, 2016). Sin embargo, en la mayoría de las propuestas se emplean criterios que han surgido desde la perspectiva de profesionales que no tuvieron en cuenta la opinión de la ciudadanía (Ewing y Clemente, 2013).

Asimismo, en el caso de las personas sin autonomía, tampoco se ha desarrollado un análisis simultáneo de los diferentes tipos de personas. Se ha encontrado que los diseños que favorecen a algunos pueden afectar los desplazamientos de otros. Por ejemplo, el diseño pensado en las personas con discapacidad motriz puede perjudicar el desplazamiento de aquellos que tienen discapacidad visual (Poldma et al., 2014). También se ha observado que usualmente se excluye del análisis a la discapacidad visual y cognitiva. En el primer caso, porque se considera que ver es un requisito para desarrollar actividades sociales (Gehl y Svarre, 2013) y, en el segundo, porque las personas con discapacidad cognitiva aún sufren discriminación social (Palacios, 2008).

Debido a que los colectivos sin autonomía no tienen un espacio diseñado para ellos y las propuestas actuales presentan deficiencias para evaluar sus necesidades, se propone el instrumento de evaluación de los entornos de proximidad inclusivos (EEPI), que ha surgido de un enfoque mixto y de la opinión de diferentes personas sin autonomía: niños; niñas; adultos mayores; personas con discapacidad (motriz, visual y cognitiva); además de cuidadores y rehabilitadores.

El artículo se organiza en cinco etapas. Primero, se desarrolla el marco teórico respectivo. Posteriormente, se señala la metodología. Luego, se presenta el método EEPI. Después, se discuten los principales hallazgos. Y finalmente, se presentan las conclusiones. 


\section{Marco teórico}

\subsection{La persona diversa: el actual sujeto de estudio de la movilidad}

A lo largo del siglo XX, las ciudades han experimentado cambios socioeconómicos y territoriales que han determinado la evolución conceptual y metodológica de la movilidad (Miralles-Guasch y Cebollada, 2009). En su primera etapa de evolución, se priorizó la circulación del automóvil, la demanda del transporte (Radelat, 2003) y los viajes que conectaban el lugar de trabajo con el de residencia (Clark et al., 2003). Asimismo, se consideró como sujeto de estudio al hombre adulto, trabajador y con excepcional capacidad física (Tonucci, 2004).

A partir de la década de los setenta, el surgimiento de los problemas energéticos y un mayor nivel de motorización pusieron en evidencia una serie de externalidades, como la congestión y la contaminación ambiental (European Commission, 2004). En esta etapa, se consideró como sujeto de estudio a los diferentes tipos de conductores y se puso énfasis en el transporte público (Clifton et al., 2007), debido a su mayor eficiencia energética y ambiental en comparación con el automóvil (Dextre y Avellaneda, 2014).

A inicios del siglo XXI, se incorporaron nuevas propuestas de organización territorial (Herce, 2009) y se reconoció que el acceso a la ciudad, los bienes y servicios es un derecho ciudadano que implica ámbitos civiles y políticos (Borja, 2010). Con esto, el sujeto de estudio de la movilidad pasó a ser la persona, con toda su diversidad (Sanz, 2005), y se incluyó en el análisis a los que no tenían autonomía, como los niños, los adultos mayores y las personas con discapacidad. También se tuvieron en cuenta las perspectivas de género y etnia (Garcia Ramon et al., 2014).

Además, a partir de la reivindicación del ser humano como sujeto de estudio de la movilidad, se ha comprendido que la calidad del espacio público influye en los patrones de movilidad y en la generación de vida urbana (Gehl y Svarre, 2013), así como en el desarrollo de los 21 nuevos derechos ciudadanos (Borja, 2013). Por ello, en la actualidad, los planes de movilidad mencionan explícitamente la transformación del espacio público. Por ejemplo, el «Plan Director de Movilidad de la Región Metropolitana de Barcelona 2020-2025» (ATM, 2019) tiene como una de sus medidas la "pacificación y ambientalización del espacio público: áreas para la ciudadanía», lo que impactará concretamente en los planes locales de movilidad.

\subsection{Diseño y evaluación de los entornos de proximidad}

El entorno de proximidad es una entidad espacial donde se articulan la ciudad y los viajes, lo que permite realizar la planificación y la evaluación de la movilidad urbana desde una perspectiva urbanística, ambiental y socioeconómica (Soria, 2011). Además, constituye el espacio principal de la vida cotidiana. De acuerdo con Talavera-García et al. (2014), habría cinco tipos de entornos de movilidad, dentro de los cuales el de proximidad es el que se relaciona- 
ría con la accesibilidad (Banister, 2011), la compacidad (OECD, 2012), los microdesplazamientos y la caminata (Miralles-Guasch y Marquet Sardà, 2013).

La reivindicación de la persona diversa, como sujeto de estudio de la movilidad, ha determinado diferentes propuestas para diseñar y evaluar los entornos. Estas se enfocan en 1) quienes frecuentan el espacio público y 2) en perspectivas inclusivas, como las de género (Garcia Ramon et al., 2014) y autonomía (Cabrera, 2019). Según quien frecuente el espacio público, puede haber dos aproximaciones. Una está basada en observación del entorno y el uso de parámetros cuantitativos. Por ejemplo, se encuentran las propuestas que indican que el entorno debe contar con seguridad vial: real y subjetiva (Sorensen y Mosslemi, 2009; Elvik et al., 2008); con confort (Fruin, 1971), y con accesibilidad, seguridad, confort y atractivo (Pozueta et al., 2009; Talavera-García et al., 2014). La segunda aproximación se basa en la observación del entorno y el uso de parámetros mixtos. Abarca la necesidad de la seguridad ciudadana (Forrester y Snell, 2007); aspectos como imageability, enclousure, escala humana, transparencia y complejidad que surgieron de la opinión de urbanistas y arquitectos (Ewing y Clemente, 2013), así como doce criterios de calidad que combinan aspectos medibles y subjetivos (Gehl, 2014) y que han sido empleados en la recuperación del espacio público en ciudades como Nueva York y Copenhague (Gehl y Svarre, 2013). Sin embargo, estas propuestas se basan en la observación de las personas que frecuentan el espacio público, por lo que no incluyen a las personas con discapacidad, que mayormente permanecen en sus hogares (Cabrera, 2019). Asimismo, resaltan el hecho de que a través de la vista se recolecta el 90\% de la información del entorno (Zimmermann, 1989), que es necesaria para el disfrute y la socialización. Esto excluye a las personas con discapacidad visual.

En el caso de los diseños desde la perspectiva inclusiva de género, se resalta el rol de las mujeres dentro de la planificación urbana y la necesidad de seguir procesos participativos. Por ejemplo, Ortiz Escalante y Gutiérrez Valdivia (2015) señalan cómo la organización Col-lectiu Punt 6 ha desarrollado herramientas para apoyar el empoderamiento de la mujer y la transformación urbana en ciudades como Barcelona y Buenos Aires. Sus herramientas buscan dar voz a las mujeres, por lo que emplean workshops, caminatas exploratorias, mapeos, auditorías de seguridad, ocupación de las calles, entre otras actividades.

En el caso de las personas sin autonomía, los diseños obedecen a los criterios surgidos de la opinión de profesionales o de experimentos en laboratorios, como el Pedestrian Accessibility Mobility Environment Laboratory (PAMELA), de la University College London. Por ejemplo, se estudió el ancho mínimo útil de una superficie podotáctil (Childs et al., 2010) y el efecto de la pendiente transversal en la fuerza que se debe aplicar al empujar una silla de ruedas (Holloway et al., 2010). Sin embargo, en la actualidad aún existen dos aspectos por resolver: si los diseños consideran las necesidades de los diferentes grupos en su conjunto (Poldma et al., 2014; Argyropoulos y Kanari, 2015; Swaine et al., 2014) y si las personas sin autonomía piensan que esta nueva 
infraestructura facilita realmente sus desplazamientos (Parkin y Smithies, 2012; Hammond y Musselwhite, 2012). Precisamente, en este artículo se presenta un instrumento que busca cumplir con ambos aspectos.

Del mismo modo, los desplazamientos de las personas adultas, en los entornos de proximidad, son evaluados mediante modelos de microsimulación peatonal (PTV, 2013). Estos consideran que un individuo puede interactuar con otros y con el entorno (Sumpter, 2006). Entre los diferentes modelos, resalta el de la fuerza social, el cual señala que las fuerzas sociopsicológicas son las que definen el comportamiento humano (Lewin, 1951). Y emplea parámetros cuantitativos como la velocidad y la densidad (TRB, 2010). Sin embargo, aún falta considerar en estos modelos las características de las personas sin autonomía. Este es otro aspecto que cubre el instrumento EEPI.

\section{Metodología}

La investigación que permitió obtener el instrumento EEPI se inició con la siguiente pregunta: ¿qué criterios podrían considerarse para elaborar un instrumento de evaluación de entornos de proximidad, de acuerdo con las necesidades de las personas sin autonomía? Para responderla, se estableció un enfoque mixto concurrente con mayor peso cualitativo, del tipo "CUALcuan» (Johnson y Christensen 2012), y se desarrolló el proceso de triangulación (Rincón et al., 1995) con investigación documental, observación, entrevistas, cuestionarios y mediciones de variables cuantitativas.

\subsection{Casos de análisis y área de estudio}

Se estudió a niños y niñas, adultos mayores, adultos con discapacidad (motriz, visual y cognitiva) y a cuidadores y rehabilitadores (fuente indirecta). Se consideró a ambos géneros y a los que nacieron con la discapacidad o la adquirieron posteriormente. Por otro lado, las unidades de análisis se seleccionaron mediante un muestreo por cadena o bola de nieve. Y debido a que no se pudo encontrar a todos los casos de análisis en un solo lugar, se escogieron seis áreas de estudio en Lima.

La plaza Diez Canseco se seleccionó porque es frecuentada por adultos mayores que caminan solos o emplean andadores, bastones y sillas de ruedas. También el colegio San Pedro porque pertenece a una zona de Lima con índice de desarrollo humano bajo, los niños iban solos al colegio y se consiguió el permiso para entrevistarlos. La Pontificia Universidad Católica del Perú porque es el centro de labores de uno de los autores y se observó a jóvenes con discapacidad y se tuvo acceso a sus familiares. Además, el colegio para personas con discapacidad cognitiva CEBE $\mathrm{N}^{\circ} 09$ porque se consiguió el permiso para realizar recorridos con los estudiantes y entrevistar a los rehabilitadores. La Unión Nacional de Ciegos del Perú porque se obtuvo acceso a sus integrantes. Y la Asociación de Talleres Protegidos de Monterrico ATAPROM porque se consiguió el permiso para entrevistar a adultos con discapacidad cognitiva leve. 
Estas áreas pertenecen a los distritos de Jesús María, El Agustino, San Miguel, Lima y Surco, respectivamente.

\subsection{Construcción del instrumento EEPI}

El instrumento EEPI fue elaborado a partir de información obtenida con las distintas técnicas de investigación descritas anteriormente. En la figura 1, se muestra la relación entre los componentes de sus dos etapas (diagnóstico y gestión) y las técnicas que les dieron origen. En el apartado 4, se brindan mayores detalles del instrumento EEPI.

\subsubsection{Etapa de diagnóstico}

EEPI cuenta en su primera etapa — de diagnóstico- con listas de chequeo conformadas por aspectos y criterios que se obtuvieron a partir de investigación documental y un enfoque cualitativo de diseño fenomenológico.

La investigación documental abarcó la búsqueda sistemática, localizada, asistémica y derivada (Avellaneda, 2007). La búsqueda sistemática utilizó bases de datos para acceder a revistas científicas periódicas. En la búsqueda localizada, se visitaron bibliotecas físicas y virtuales. Con la asistémica, se obtuvo información sin seguir ningún plan de búsqueda preconcebido; se halló material audiovisual de fundaciones y medios periodísticos, así como publicaciones en Facebook de organizaciones de personas con discapacidad. Toda esta información fue transcrita para su posterior análisis. Finalmente, la búsqueda derivada consistió en utilizar las referencias presentadas en las publicaciones consultadas.

En el enfoque cualitativo de diseño fenomenológico se realizaron entrevistas a profundidad para conocer las experiencias y perspectivas de las personas a través de sus propias voces (Hernández-Sampieri et al., 2014). Las entrevistas fueron flexibles, dinámicas (King y Horrocks, 2010) e individuales y siguieron un esquema semiestructurado. En el caso de los niños y las niñas, se realizó una

Figura 1. Componentes de EEPI y técnicas de recolección

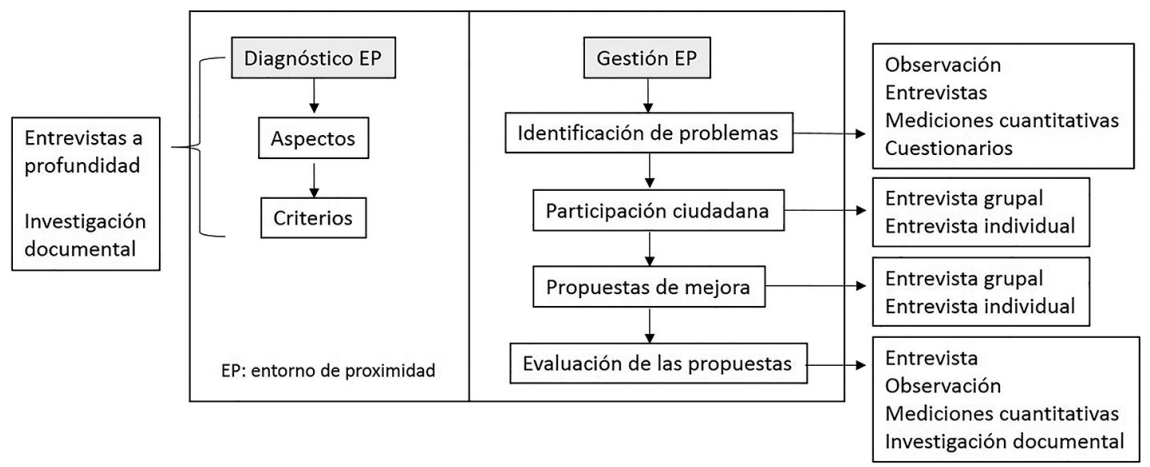

Fuente: elaboración propia. 
Figura 2. Proceso de análisis de la información cualitativa

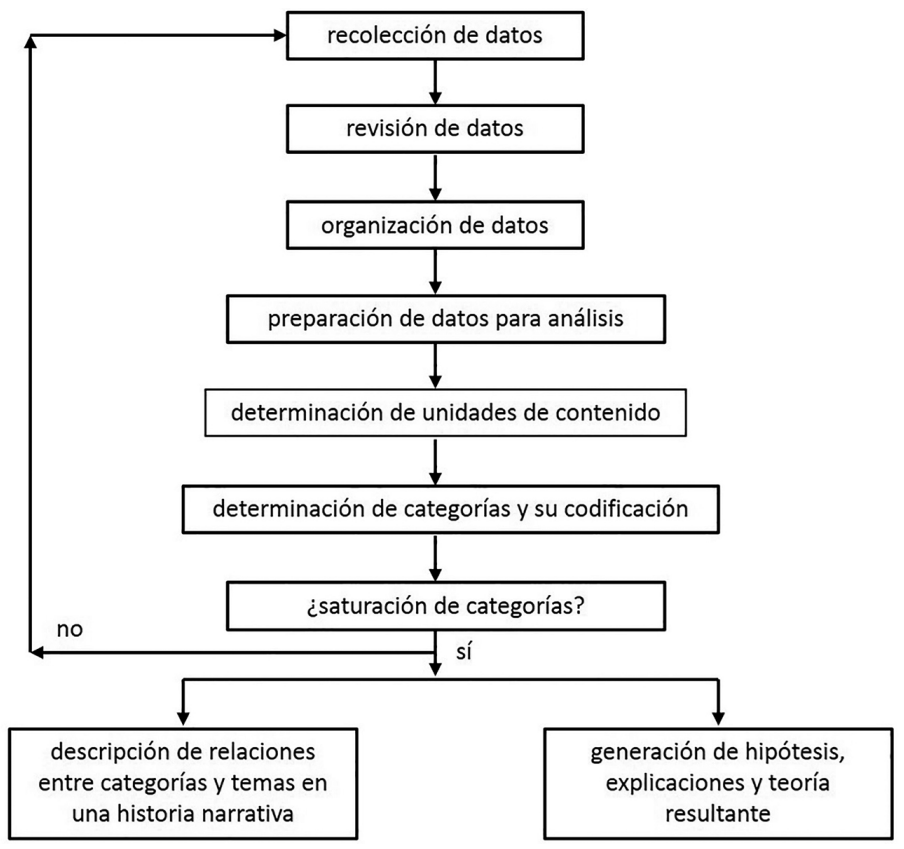

Fuente: elaboración propia.

entrevista grupal a siete de ellos, debido a que así lo estipulaba el permiso obtenido. El muestreo se realizó mediante cadena o bola de nieve. Participaron 32 personas: siete niños y niñas (entre 8 y 12 años); cinco adultos mayores (entre 60 y 93 años, que se desplazaban solos o con la ayuda de algún elemento de apoyo como un andador, bastón o sillas de ruedas); seis personas adultas con discapacidad motriz (silla de ruedas y muletas); siete con discapacidad visual (congénita y con residuo visual), cuatro con discapacidad cognitiva (autismo, síndrome de Down y lesión cerebral de nivel leve) y tres cuidadores (familiares y terapeutas). Las entrevistas fueron anónimas y tuvieron una duración de entre 45 y 65 minutos, salvo en el caso de las personas con discapacidad cognitiva y adultos mayores, en que las entrevistas duraron alrededor de 30 minutos. Esto se debió al cansancio o la pérdida de la atención de los participantes. Asimismo, se necesitó contar con una guía de entrevista y con la presencia de alguno de los familiares de las personas con discapacidad cognitiva para que reformularan las preguntas, con un código de comunicación que solo ellos conocen. En la figura 2, se presenta el proceso de análisis de la información cualitativa.

El proceso de análisis se inició con la revisión y la organización de los datos y la transcripción literal de las entrevistas. Luego se determinaron las unidades de contenido (flujo libre). En el software NVIVO 11 se examinó el significado de las unidades y, mediante las técnicas de escrutinio, procesamiento y 
comparación constante, se determinaron las categorías (Hernández-Sampieri et al., 2014; Tracy, 2013). Posteriormente, se decidió que no era necesario recolectar más información cuando se alcanzó la saturación (Neuman, 2009). Finalmente, se establecieron explicaciones a partir de las inferencias y metainferencias obtenidas, lo que dio origen a los aspectos y criterios que conforman las listas de chequeo de EEPI.

\subsubsection{Etapa de gestión}

Mediante la información recolectada con la observación, cuestionarios, entrevistas a profundidad, investigación documental y mediciones de variables cuantitativas, se estableció la etapa de gestión del instrumento EEPI, que abarca cuatro pasos (figura 1). El paso 1, de identificación de problemas, se construyó a través de entrevistas a profundidad (ver 3.2.1), observación y cuestionarios. La observación realizada fue directa o pasiva para no influir en el comportamiento de las personas. Se enfocó en las interacciones sociales y en los desplazamientos de las personas. Para ello, se emplearon libreta de notas, la vista (naked eye) y registro fotográfico, recomendados y descritos por Gehl y Svarre (2013). Asimismo, a través de un muestreo no probabilístico, se realizaron 105 cuestionarios, con preguntas cerradas y abiertas, a adultos mayores en la plaza San José. Se recabó información sobre la frecuencia de sus visitas a la plaza, los problemas a que se enfrentaron al desplazarse desde su casa y los factores que les favorecen. Luego, los pasos 2 y 3 , de participación ciudadana y propuestas de mejora, se determinaron a partir de la información obtenida con las entrevistas a profundidad, individuales y grupales (ver 3.2.1).

Por último, el paso 4, de evaluación de las propuestas de mejora, se estableció de acuerdo con lo hallado en las entrevistas a profundidad, observación, investigación documental y medición de variables cuantitativas. En este último caso, se registraron, en las seis áreas de estudio, las trayectorias y la configuración de peatones mediante las técnicas de recorrido y seguimiento, planteadas y descritas por Gehl y Svarre (2013). También se midieron velocidades y aceleraciones. Para ello, se adoptó un diseño no experimental con enfoque transversal y alcance exploratorio (Hernández-Sampieri et al., 2014). El proceso consistió en filmar con un dron (Pantom 2) los desplazamientos de las personas para luego obtener las velocidades y aceleraciones (Weidmann, 1993). Fue indispensable determinar el tamaño mínimo de muestra, para lo cual se utilizó un nivel de confianza del $95 \%$ y un error máximo admisible del $10 \%$ de la media. Además, se empleó la estadística descriptiva, pruebas paramétricas ( $t$ student y ANOVA), de hipótesis y de bondad de ajuste (Kolmogorov-Smirnov y Chi Cuadrado). Con la información obtenida, se determinaron once criterios cuantitativos (ver tabla 3) que permiten evaluar la calidad de los desplazamientos de las personas sin autonomía.

\section{El instrumento EEPI}

El instrumento de evaluación de los entornos de proximidad inclusivos (EEPI) puede ser empleado en ciudades con características parecidas a Lima y abar- 
Figura 3. Instrumento de evaluación de entornos de proximidad (EP) inclusivos

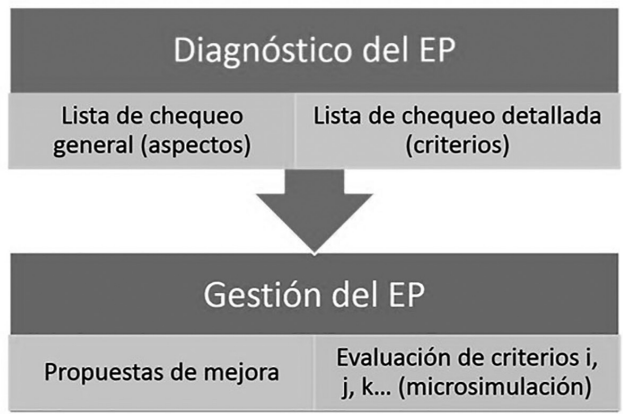

Fuente: elaboración propia.

ca edificaciones, aceras, plazas y cruceros peatonales. El esquema general del instrumento se muestra en la figura 3, donde se aprecian dos etapas de evaluación. La primera corresponde a una situación de diagnóstico, mientras que la segunda a una operacional que está ligada a la gestión del entorno y al uso de herramientas como la microsimulación peatonal. Cabe mencionar que se puede aplicar solo la etapa de diagnóstico o ambas.

\subsection{Evaluación de diagnóstico}

En esta primera etapa, se emplea una lista de chequeo general y una detallada. Ambas fueron elaboradas a partir de las opiniones de las personas que participaron en la investigación. La lista de chequeo general abarca diez aspectos (tabla 1) que son resultado del análisis de unidades y categorías (ver 3.2.1). Se agrupan en tres ámbitos: personal, físico y social. El ámbito personal comprende el clima, los sentidos humanos y la navegación. El físico incluye las

Tabla 1. Lista de chequeo general de EEPI

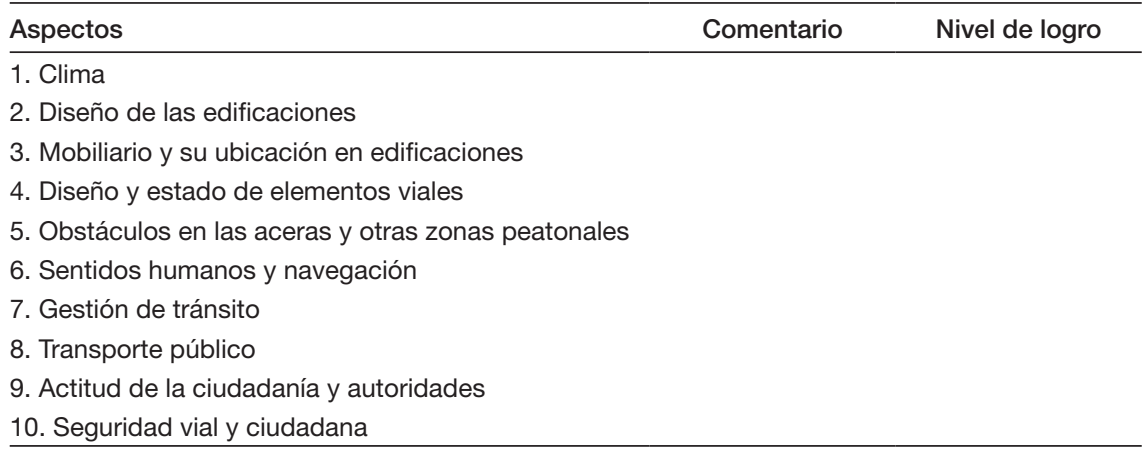

Fuente: elaboración propia. 
consideraciones de diseño de las edificaciones; el mobiliario y su ubicación en los edificios; el diseño y estado de elementos viales; los obstáculos en las aceras y otras zonas peatonales; la gestión de tránsito, y el transporte público. Finalmente, el ámbito social abarca la actitud de la ciudadanía y autoridades, y la seguridad vial y ciudadana. Además se pueden realizar comentarios y registrar el nivel de logro percibido.

Con la lista de chequeo detallada, se evalúan los criterios que conforman cada uno de los diez aspectos generales (tabla 1). A continuación, se detallan los aspectos y sus criterios.

- Clima: se considera el efecto de las lluvias y el calor en la infraestructura para las personas sin autonomía y en sus ayudantes.

- Diseño de las edificaciones: se analizan aspectos relacionados con la geometría de las puertas y pasillos; tipos de ascensores; plataformas de elevación mecánica; geometría de las escaleras; calidad de las superficies para caminar; dispositivos que brinden información no visualmente orientada; baños para discapacitados; ubicación de elementos como percheros, llaves de luz y espejos; rutas y protocolos de evacuación.

- Mobiliario y su ubicación en las edificaciones: se consideran la calidad y la ubicación de las mesas, carpetas, ventanillas, etcétera. También se identifica si los pasillos de las edificaciones se encuentran bloqueados por objetos.

- Diseño y estado de los elementos viales: se evalúan la orientación, ancho, estado y pendiente de las rampas; el ancho efectivo, pendientes y estado de las aceras; geometría de las medianas; desnivel entre la acera y calzada; geometría de los puentes peatonales y sus barandas; la calidad de la calzada; el diseño de la vía; la longitud de cruce en las esquinas; distinción entre la entrada a los pasajes y las intersecciones, y la calidad del sistema de desagüe.

- Obstáculos en las aceras y otras vías peatonales: se examinan los obstáculos a nivel, como postes de luz, vehículos estacionados, tapas de buzones, etcétera; los obstáculos elevados sobre la acera, como puertas de garajes, ramas de árboles, ventanas abiertas, tubos de quioscos, etcétera; la ubicación de los obstáculos, y la cantidad de mascotas que se desplazan por las aceras y plazas.

- Sentidos humanos y navegación: abarca criterios como el brillo solar; niveles de ruido; magnitud del flujo peatonal; niveles de contaminación y olores desagradables; la iluminación nocturna; referencias arquitectónicas y naturales; restaurantes o servicios que se puedan utilizar como referencia para navegar; rutas que tengan espacios para descansar y que brinden sombra; cantidad de información que se debe procesar para cruzar las calles, y dispositivos que brinden información no visualmente orientada.

- Gestión del tráfico: se analizan la magnitud y control de las velocidades; la regulación de la circulación de mototaxis (vehículo para dos pasajeros) y vehículos pesados; el diseño de los tiempos de los semáforos y su tipo, y la calidad, diseño e iluminación de las señales de tránsito.

- Transporte público: se consideran el diseño de los autobuses, coasters (vehículo para 26 pasajeros) y combis (vehículo para 11 pasajeros); la can- 
tidad de asientos especiales y la información de sus rutas; la cantidad de personas en los autobuses; el comportamiento de los cobradores de autobuses y el sistema de información en las paradas; la presencia de cinturones en los autobuses para personas sin autonomía; el diseño y características de los taxis y servicios especiales.

- Actitud de la ciudadanía y autoridades: se analiza si los conductores de vehículos y peatones insultan a las personas sin autonomía; si los taxistas recogen a estas personas; si los choferes de transporte público y de taxis bloquean los cruceros peatonales; si los choferes de transporte público inician la marcha cuando estas personas aún están subiendo; si los choferes de mototaxis se desplazan por rutas permitidas; si se observa a peatones, choferes y cobradores de transporte público ayudar o dar información a las personas sin autonomía. También si los peatones que usan los puentes peatonales empujan a las personas sin autonomía, si los conductores de automóviles se estacionan en lugares para discapacitados, si las autoridades locales incorporaron a las personas sin autonomía en el diseño del espacio público, entre otros aspectos.

- Seguridad vial y ciudadana: se evalúa si los vehículos al girar ceden el paso a los peatones; el efecto del nivel de tráfico vehicular en las personas y en los accidentes; la vigilancia contra robos; la percepción social de que ciertos conductores de mototaxis son delincuentes, y la realización de actividades en los barrios.

La lista de chequeo detallada contiene criterios cualitativos y cuantitativos. A ambos se les asignan puntajes de cero a cinco, a través de cuatro niveles de calidad: indeseado (0-1 punto), regular ( 2 puntos), bueno ( $3-4$ puntos) y excelente (5 puntos). Los cualitativos dependen de la percepción del evaluador y los cuantitativos, como el ancho efectivo de la acera, pueden ser medidos y, de acuerdo con la tabla 2, se les asigna el puntaje. Debe mencionarse que los autores de este artículo sugieren esta escala de valoración de acuerdo con los lineamientos establecidos en el ámbito de la seguridad vial (iRAP, 2018). En el caso de que un criterio no se aplique a la situación analizada, se debe colocar en la casilla de comentarios la expresión "no evaluado (NE)».

Finalmente, al sumar los puntajes obtenidos por cada criterio, se podrían determinar el nivel de logro de cada aspecto y el nivel de calidad de la zona estudiada. Para ello, se establecen cuatro niveles de calidad, según el porcentaje de puntos obtenidos con respecto a la máxima cantidad de puntos posibles: indeseado (menor del $40 \%$ ), regular (entre el $40 \%$ y el $70 \%$ ), bueno (entre el $70 \%$ y el $90 \%$ ) y excelente (entre el $90 \%$ y el $100 \%$ ). La lista de chequeo detallada y una mayor información de la valoración de los criterios se pueden encontrar en Cabrera (2019: 366).

\subsection{Gestión del entorno de proximidad}

La gestión del entorno abarca al ámbito operacional (diseño y evaluación de las zonas peatonales) que tradicionalmente ha sido desarrollado por la ingeniería. 
Tabla 2. Niveles de calidad de criterios cuantitativos

\begin{tabular}{|c|c|c|c|c|}
\hline Criterio & Excelente & Bueno & Regular & Indeseado \\
\hline Ancho de acera $(m)^{1}$ & $>3$ & $1,8-3$ & $1,2-1,8$ & $<1,2$ \\
\hline Pendiente longitudinal de aceras $(\%)^{2}$ & $<3,5$ & $3,5-5$ & $5-9$ & $>10$ \\
\hline Ruido Lden $(\mathrm{dBA})^{3}$ & $<50$ & $50-65$ & $65-70$ & $>70$ \\
\hline Ancho de rampa $(m)^{4}$ & $>1,5$ & $1,2-1,5$ & $0,9-1,2$ & $<0,9$ \\
\hline Pendiente de rampas cortas $(\%)^{2}$ & $<3,5$ & $3,5-5$ & $5-8$ & $>8$ \\
\hline $\begin{array}{l}\text { Ancho de mediana o isla de refugio peatonal, } \\
\text { en la dirección de cruce de la calle }(\mathrm{m})^{5}\end{array}$ & $>2,4$ & $2,4-2$ & $1,5-2$ & $<1,5$ \\
\hline Ancho de puertas y pasillos $(m)^{4}$ & $>1,5$ & $1,2-1,5$ & $1,0-1,2$ & $<1$ \\
\hline Número de carriles a cruzar en la intersección $(\mathrm{m})^{5}$ & 1 & 2 & 3 & $>3$ \\
\hline $\begin{array}{l}\text { Flujo peatonal en aceras o vías peatonales } \\
\text { (peatones } / \mathrm{min} / \mathrm{m})^{6}\end{array}$ & $<16$ & $16-33$ & $33-49$ & $>49$ \\
\hline Flujo vehicular (veh/h) ${ }^{7}$ & 200 & $200-550$ & $550-1900$ & $>1900$ \\
\hline $\begin{array}{l}\text { Velocidad de los vehículos al colisionar con un } \\
\text { peatón en la zona de conflicto }(\mathrm{km} / \mathrm{h})^{8} \text {. Se considera } \\
\text { que la velocidad de circulación estaría cerca de esta } \\
\text { velocidad de colisión }\end{array}$ & 20 & $20-30$ & $30-40$ & $40-80$ \\
\hline
\end{tabular}

Fuente: adaptado de las siguientes referencias:

1. De Talavera-García et al., (2014), según Manchon y Santamera (1995); Prinz (1986) y Sanz (2008)

2. Adaptado de Sanz (1996)

3. Adaptado de EEA (2010)

4. Adaptado de Huerta (2007)

5. Adaptado de NACTO (2013)

6. Adaptado de TRB (2000)

7. Adaptado de Appleyard et al. (1981)

8. Adaptado de Bonanomi (1990), según la probabilidad de fallecimiento del peatón en la colisión

Como se mencionó anteriormente, se deben desarrollar cuatro pasos: 1) la identificación de problemas, 2) la participación ciudadana, 3) las propuestas de mejora y 4) la evaluación de propuestas (ver 3.2.2). Debe resaltarse que estos pasos se derivan de esta investigación, pero algunos de los instrumentos que se presentan son sugeridos por los autores de este artículo. Por ejemplo, de las opiniones de las personas se concluyó que para identificar problemas es importante observar el entorno, pero las personas no indicaron específicamente qué instrumento se debe utilizar para cumplir con este fin.

Antes de describir los pasos de la gestión del entorno, debe mencionarse que a partir de este momento la palabra sugiere hace referencia a las recomendaciones de los autores de este artículo (a partir de investigación documental y prácticas profesionales actuales) y no es un resultado directo de la investigación de campo.

El primer paso —identificación de problemas - implica realizar tres actividades: 1) recolectar datos, 2) aplicar la lista de chequeo detallada y 3) crear un mapa de barreras. Para recolectar datos, primeramente se debe observar el entorno; para ello, se sugiere utilizar una bitácora y un registro fotográfico (Gehl y Svarre, 2013). Otros datos relevantes son los flujos peatonales y vehiculares, la geometría de los elementos viales y los ciclos y fases de los 
Figura 4. Esquema general de la gestión del entorno (análisis operacional)

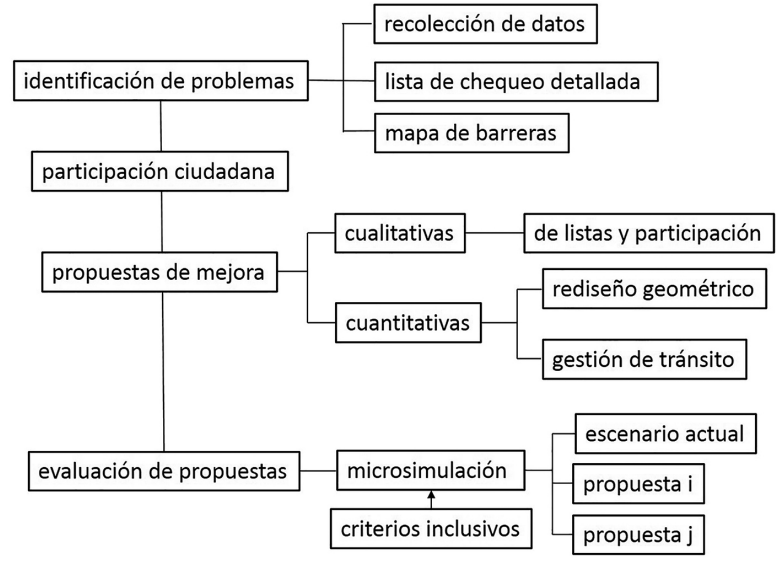

Fuente: elaboración propia.

semáforos. Para medir los flujos vehiculares y peatonales, se sugiere emplear los procedimientos del TRB (2010). Asimismo, se sugiere obtener la geometría de los elementos viales mediante levantamientos topográficos planialtimétricos y los tiempos del semáforo mediante el uso de cronómetros.

En la segunda actividad, se utilizan listas de chequeo detalladas (ver 4.1) y, en la tercera, se elaboran mapas de barreras, donde se señala la ubicación de los obstáculos y las zonas de conflicto que surgen de la interacción de las personas y vehículos. Se sugiere utilizar el plano topográfico obtenido anteriormente en la primera actividad o, en su defecto, un bosquejo.

Posteriormente, en el segundo paso, se impulsa la participación del vecindario. Para ello, se sugiere utilizar herramientas cualitativas, como entrevistas individuales o grupales y focus groups, con el fin de recoger información que se complemente y contraste con la de otras técnicas. Después, en el tercer paso, se presentan propuestas de mejora que se enfocan en las deficiencias geométricas, el estado de los elementos viales, el control del tránsito y la seguridad vial.

Finalmente, en el cuarto y último paso —evaluación de propuestas—, se compara la situación actual con las propuestas de mejora. Para determinar los posibles beneficios, se sugiere incorporar los criterios cuantitativos (globales y específicos) identificados en esta investigación en algún software de microsimulación. Los criterios globales (que consideran el rendimiento promedio en todo el entorno) abarcan el tiempo de viaje, la velocidad, la densidad y la demora. Dentro de los específicos, se incluyen siete criterios para el análisis exclusivo de personas sin autonomía: el factor de uso de las rampas, el factor de uso de calzada, el grado de obstaculización, los conflictos producidos entre los diferentes modos, el factor sensorial, el índice de seguridad y el flujo de información. En la tabla 3, se muestran todos los criterios mencionados, así como su definición y expresión matemática (ecuación). 
Tabla 3. Criterios propuestos para la microsimulación peatonal

\begin{tabular}{|c|c|c|}
\hline Criterio & Definición & Ecuación \\
\hline Tiempo de viaje (s) & $\begin{array}{l}\text { Valor promedio de los tiempos de viaje de todos los } \\
\text { peatones, o por tipo de peatón, desde su origen hasta } \\
\text { su destino }\end{array}$ & $\begin{array}{l}\left(\mathrm{T}_{\mathrm{i}}\right) / \mathrm{n} \\
\mathrm{T}_{\mathrm{i}} \text { : tiempo de viaje total individual } \\
\mathrm{n} \text { : número de peatones }\end{array}$ \\
\hline $\begin{array}{l}\text { Densidad } \\
\text { (peatones/m²) }\end{array}$ & $\begin{array}{l}\text { Cantidad de peatones por metro cuadrado, en una o } \\
\text { varias secciones definidas por el usuario. Se obtendría } \\
\text { su distribución y otros parámetros estadísticos }\end{array}$ & $\begin{array}{l}\mathrm{n} / \mathrm{A} \\
\mathrm{n}: \text { número de peatones } \\
\mathrm{A} \text { : área delimitada }\end{array}$ \\
\hline Velocidad (m/s) & $\begin{array}{l}\text { Valor promedio de las velocidades instantáneas de } \\
\text { todos los peatones o tipo de peatón (al cruzar una o } \\
\text { varias secciones definidas por el usuario) }\end{array}$ & $\begin{array}{l}\left(\mathrm{V}_{\mathrm{i}}\right) / \mathrm{n} \\
\mathrm{V}_{\mathrm{i}} \text { : velocidad individual } \\
\mathrm{n} \text { : número de peatones }\end{array}$ \\
\hline Demora (s/peatón) & $\begin{array}{l}\text { Valor promedio de todos los peatones, o tipo de } \\
\text { peatón, que resulta de comparar el tiempo de viaje } \\
\text { en la ruta real versus el tiempo en la ruta deseada, } \\
\text { con condiciones favorables }\end{array}$ & $\begin{array}{l}{\left[\left(\mathrm{T}_{\mathrm{i}}-\mathrm{T}_{\mathrm{Vd}}\right)\right] / \mathrm{n}} \\
\mathrm{T}_{\mathrm{i}}: \text { tiempo de viaje total individual } \\
\mathrm{T}_{\mathrm{Vd}} \text { : tiempo de viaje individual en ruta deseada } \\
\mathrm{n} \text { : número de peatones }\end{array}$ \\
\hline $\begin{array}{l}\text { Factor de uso } \\
\text { de rampas (\%) }\end{array}$ & $\begin{array}{l}\text { Indica el porcentaje de rampas, en una zona determi- } \\
\text { nada por el usuario, que pueden utilizarse debido a su } \\
\text { ancho y pendiente adecuados }\end{array}$ & $\begin{array}{l}\left(\mathrm{N}_{\mathrm{ru}} / \mathrm{N}_{\mathrm{tr}}\right) \\
\mathrm{N}_{\mathrm{ru}}: \text { número de rampas utilizables } \\
\mathrm{N}_{\mathrm{tr}}: \text { número total de rampas }\end{array}$ \\
\hline $\begin{array}{l}\text { Factor de uso de } \\
\text { la calzada (\%) }\end{array}$ & $\begin{array}{l}\text { Es el cociente del tiempo de viaje en la calzada } \\
\text { y el tiempo de viaje total. Es representativo de } \\
\text { las personas en silla de ruedas. Con el modelo } \\
\text { se obtendría la distribución de dicho factor de } \\
\text { uso y otros parámetros estadísticos }\end{array}$ & $\begin{array}{l}{\left[\mathrm{Si} \mathrm{w}_{\text {ea }}<\mathrm{w}_{\min } \text { o rampa es inadecuada] }\right.} \\
\mathrm{T}_{\mathrm{Vc}} / \mathrm{T}_{\mathrm{i}} \\
\mathrm{W}_{\text {ea }}: \text { ancho efectivo acera } \\
\mathrm{W}_{\text {min }}: \text { ancho mínimo acera } \\
\mathrm{T}_{\mathrm{V}}: \text { tiempo de viaje individual en la calzada } \\
\mathrm{T}_{\mathrm{i}}: \text { tiempo de viaje total individual }\end{array}$ \\
\hline $\begin{array}{l}\text { Grado de } \\
\text { obstaculización }\end{array}$ & $\begin{array}{l}\text { Es el producto del tiempo de viaje y el número de } \\
\text { obstáculos observados en una sección definida por el } \\
\text { usuario. Los obstáculos pueden ser a nivel o elevados. } \\
\text { Puede ser empleado para diferentes peatones, pero } \\
\text { principalmente, para personas con discapacidad } \\
\text { visual. Se obtendrían la distribución de dicho factor } \\
\text { y otros parámetros estadísticos }\end{array}$ & $\begin{array}{l}\mathrm{T}_{i}{ }^{*} \mathrm{~N} \\
\text { Ti: tiempo de viaje en la sección definida } \\
\mathrm{N} \text { : número de obstáculos en la sección definida }\end{array}$ \\
\hline $\begin{array}{l}\text { Coeficiente de } \\
\text { conflicto modal }\end{array}$ & $\begin{array}{l}\text { Mide la interacción entre el flujo vehicular y } \\
\text { bicicletas con el flujo peatonal. Considera la } \\
\text { velocidad promedio, la cantidad de vehículos } \\
\text { y el número de peatones que estarían potencialmente } \\
\text { involucrados en los conflictos }\end{array}$ & $\begin{array}{l}\left(\mathrm{V}_{\mathrm{pv}}{ }^{*} \mathrm{~N}_{\mathrm{v}}+\mathrm{V}_{\mathrm{pb}}{ }^{*} \mathrm{~N}_{\mathrm{b}}\right)^{\star} \mathrm{n} \\
\mathrm{V}_{\mathrm{p}} \text { : velocidad promedio de vehículos } \\
\mathrm{N}_{\mathrm{v}} \text { : número de vehículos (coches, autobuses, } \\
\text { camiones etc.) } \\
\mathrm{V}_{\mathrm{pb}} \text { : velocidad promedio de bicicletas } \\
\mathrm{N}_{\mathrm{b}} \text { : número de bicicletas } \\
\mathrm{n} \text { : número de peatones }\end{array}$ \\
\hline Factor sensorial & $\begin{array}{l}\text { Mide el efecto del ruido, la temperatura y la } \\
\text { variedad arquitectónica en las personas con } \\
\text { discapacidad o en los peatones en general }\end{array}$ & $\begin{array}{l}\left(\mathrm{V}_{\mathrm{i}} \mathrm{N}_{\mathrm{fd}}\right) /\left(\mathrm{N}_{\text {ruido }}{ }^{*} \mathrm{~T}\right) \\
\mathrm{V}_{\mathrm{i}}: \text { velocidad de persona con discapacidad } \\
\text { Nfd: número de fachadas diferentes en la cuadra } \\
\mathrm{N}_{\text {ruido }}: \text { nivel de ruido } \\
\mathrm{T}: \text { temperatura }\end{array}$ \\
\hline $\begin{array}{l}\text { Índice de } \\
\text { seguridad }\end{array}$ & $\begin{array}{l}\text { Valor promedio de las veces en que los peatones } \\
\text { invaden la zona de seguridad de las personas con dis- } \\
\text { capacidad, en una sección determinada por el usuario. } \\
\text { Puede obtenerse por tipo de usuario }\end{array}$ & $\begin{array}{l}\left(\mathrm{N}_{\mathrm{i}}\right) / \mathrm{n} \\
\mathrm{N}_{\mathrm{i}} \text { : número de veces que se invade la zona de } \\
\text { seguridad de un peatón con discapacidad } \\
\mathrm{n} \text { : número de peatones con discapacidad }\end{array}$ \\
\hline $\begin{array}{l}\text { Flujo de } \\
\text { información }\end{array}$ & $\begin{array}{l}\text { Se define como el producto entre el tiempo que } \\
\text { se necesita para cruzar la calle, en las esquinas, } \\
\text { y el número de señales que se pueden observar, ya } \\
\text { sean horizontales, verticales, reguladoras } \\
\text { preventivas o informativas }\end{array}$ & $\begin{array}{l}\mathrm{T}_{\mathrm{c}}{ }^{*} \mathrm{~N}_{\mathrm{s}} \\
\mathrm{T}_{\mathrm{c}}: \text { tiempo de cruce } \\
\mathrm{N}_{\mathrm{s}}: \text { número de señales }\end{array}$ \\
\hline
\end{tabular}

Fuente: elaboración propia. 
Finalmente, los criterios hallados en esta investigación se pueden incluir en los modelos de microsimulación disponibles comercialmente o en los desarrollados con fines académicos. En cualquier caso, deben cumplir con dos requisitos. Primero, deben haber sido validados, independientemente de que hayan sido creados a partir de una base teórica o empírica. Y segundo, deben ser capaces de modelar la interacción de los peatones con los vehículos motorizados y los modos de micromovilidad que están apareciendo, como skates y scooters.

\section{Discusión}

Como se mencionó en el marco teórico (ver 2.2), el diseño de entornos inclusivos se realiza siguiendo criterios que han surgido de la observación del entorno, la perspectiva de los profesionales y experimentos en laboratorio. Pero, con la finalidad de fomentar la inclusión social y diseñar espacios de acuerdo con las necesidades de las personas sin autonomía, es necesario conocer, desde su voz, cuáles son sus requerimientos. Por ello, en esta investigación se construye el instrumento EEPI para cumplir con esta necesidad.

El método EEPI surgió de la aplicación de diferentes técnicas de investigación, dentro de las cuales las entrevistas fueron fundamentales. Y se encontró que realizarlas a personas con discapacidad cognitiva puede ser un desafío. Se necesita contar con una guía de entrevista y con la presencia de alguno de sus familiares para que reformule las preguntas empleando un código especial de comunicación. Asimismo, solo fue posible trabajar con personas con discapacidad cognitiva leve, y no se pudo examinar los casos medios y graves a los que se tuvo acceso. Esta situación muestra que se necesita profundizar en los diferentes ámbitos de la discapacidad cognitiva, como la metodológica.

Igualmente, al procesar la información de las entrevistas a profundidad, se pudo apreciar que cada grupo de personas tiene diferentes características y necesidades, las que inclusive entran en conflicto. Este hallazgo confirma los resultados de otras investigaciones (Poldma et al., 2014; Los Angeles County, 2011; Lid y Solvang, 2016) y resalta la valía de EEPI al considerar a múltiples ciudadanos.

Del mismo modo, el instrumento EEPI es flexible y fácil de aplicar, ya que puede servir para planificar o gestionar los entornos. En la planificación, se emplean listas de chequeo que consideran los siguientes ámbitos: personal, físico y social. Esto lo diferencia de los instrumentos existentes que se basan en el ámbito físico. Asimismo, la asignación subjetiva de puntajes a los criterios (de 0 a 5 ) puede ser perfectible, aunque esta corresponde a las recomendaciones empleadas en el campo de la seguridad vial.

En la gestión del entorno, se requiere de una mayor especialización, al estar estrechamente ligada al uso de la microsimulación peatonal. Sin embargo, existen tres aspectos a tener en cuenta: 1) el debate sobre el uso de la microsimulación, 2) la validez matemática de los criterios cuantitativos y 3) la necesidad de incorporar más colectivos y, especialmente, la perspectiva de género. 
Sobre si la microsimulación peatonal es necesaria para evaluar los desplazamientos de los colectivos sin autonomía, se considera que actualmente es la única herramienta capaz de adaptarse para detectar los problemas que surgen de la interacción de peatones y otros modos de transporte. Además, las autoridades podrían tomar mejores decisiones al observar los fenómenos en un entorno visual (computadora). Y, finalmente, contribuiría a que los profesionales, como los ingenieros, fueran más conscientes de las necesidades de los colectivos sin autonomía al tener que considerarlos en sus modelos.

De igual forma, se reconoce que los nuevos criterios cuantitativos (globales y específicos) han surgido a partir de una investigación exploratoria, por lo que las expresiones matemáticas (tabla 3) deben ser validadas. Además, estos criterios son solo algunos de los tantos que podrían utilizarse, pero que, a juicio de los autores de este artículo, podrían estar entre los más representativos. También sería necesario determinar el intervalo de acción de cada parámetro. Esto es lo que se conoce como la estandarización de resultados o la determinación de niveles de servicio (TRB, 2010).

Igualmente, debe mencionarse que el instrumento que se propone es totalmente perfectible y que aún queda como tarea pendiente investigar los requerimientos de otros grupos sociales, como las personas que tienen dos o más discapacidades, así como el factor cultural, étnico y de género para conseguir espacios públicos accesibles.

Finalmente, debe destacarse que este instrumento, a pesar de representar las características de Lima, puede ser aplicado en ciudades con características similares. Sin embargo, no debe olvidarse que los criterios de la lista de chequeo necesitarían ser adaptados a la realidad social y ambiental de cada caso concreto. También este instrumento no reemplaza a los existentes (ver 2.2), sino que los complementa.

\section{Conclusiones}

Los resultados indican que es necesario reconocer la diversidad humana en el proceso de diseño y evaluación de los entornos de proximidad, y que los diferentes tipos de personas sin autonomía tienen diversas necesidades, las cuales también pueden entrar en conflicto. Por ello, se debe incentivar la inclusión de los ciudadanos considerando las dimensiones personal, física y social.

Asimismo, se ha detectado que dentro de las personas sin autonomía existen grupos que han sido marginados, por lo que actualmente no hay, por ejemplo, metodologías claras para entrevistar a personas con discapacidad cognitiva $o$ investigación sobre sus características de desplazamiento y las barreras que enfrentan.

De igual forma, las personas analizadas manifestaron que el proceso de diseño se relaciona con una cadena de accesibilidad que abarca los edificios, los entornos de proximidad y el transporte público. Esto sugiere la necesidad de que las políticas públicas apliquen una mirada holística a las estrategias de inclusión social. 
Además, el instrumento EEPI presenta ocho aportaciones principales: 1) incorpora a diversas personas sin autonomía para evaluar los espacios urbanos; 2) se basa en la voz de los colectivos analizados y no en las opiniones de los profesionales; 3) combina metodologías cualitativas y cuantitativas; 4) no solo considera criterios físicos, sino también personales y sociales; 5) puede ser empleado en edificaciones, aceras, plazas y cruceros peatonales; 6) brinda la opción de realizar la planificación del entorno o su gestión; 7) es un instrumento útil porque es fácil de aplicar y transferir, y 8) no reemplaza a los instrumentos existentes sino que los complementa.

A pesar de las múltiples aportaciones, el instrumento EEPI es totalmente perfectible y puede ampliarse $\mathrm{u}$ optimizarse para integrar otros aspectos como el cultural, el étnico y de género, con los que se podrían conseguir entornos más accesibles.

\section{Referencias bibliográficas}

APPLEYARD, Donald (1981). Liveable streets. Berkeley: University of California Press. ARgYropoulos, Vasillios y KanarI, Charikleia (2015). «Re-imagining the museum through "touch": reflections of individuals with visual disability on their experience of museum-visiting in Greece». ALTER, 9, 130-143. $<$ https://doi.org/10.1016/j.alter.2014.12.005>

ATM (2019). «Plan Director de Movilidad de la Región Metropolitana de Barcelona (2020-2025)». Recuperado de <https://www.atm.cat/web/ca/document_ pdm_2020.php> [Fecha de consulta: 12 de febrero de 2020].

Avellaneda, Pau (2007). Movilidad, pobreza y exclusión social. Un estudio de caso en la ciudad de Lima. Tesis de doctorado. Bellaterra: Universitat Autònoma de Barcelona.

BANISTER, David (2011). «The trilogy of distance, speed and time». Journal of Transport Geography, 19 (4), 950-959. $<$ https://doi.org/10.1016/j.jtrangeo.2010.12.004>

BONANOMI, Lidya (1990). Le temps des rues. Vers un nouvel aménagement de l'espace rue. Lausana: IREC Institut de Recherche sur l'Environnement Construit, Ecole Polytechnique Fédérale de Lausanne, GCR Groupe Conseil Romand pour la Modération de la Circulation.

BORJA, Jordi (2010). La ciudad conquistada. Segunda reimpresión. Madrid: Alianza Editorial.

- (2013). Revolución urbana y derechos ciudadanos. Madrid: Alianza Editorial.

CABRERA, Félix (2019). Movilidad, espacio público y ciudadanos sin autonomía: el caso de Lima. Tesis de doctorado. Bellaterra: Universitat Autònoma de Barcelona. Recuperado de <https://ddd.uab.cat/pub/tesis/2019/hdl_10803_667392/icv1de1.pdf>.

CHILDS, Craig; THOMAS, Carol; SHARP, Sue y TYLER, Nick (2010). Can shared surfaces be safely negotiated by blind and partially sighted people? TRANSED 2010. Hong Kong.

Clark, William; HuANGB, Youqin y WitherSC, Suzanne (2003). «Does commuting distance matter? Commuting tolerance and residential change». Regional Science and Urban Economics, 33, 199-221. $<$ https://doi.org/10.1016/S0166-0462(02)00012-1>

Clifton, Kelly; Livi SMith, Andréa y Rodriguez, Daniel (2007). «The development and testing of an audit for the pedestrian environment». Landscape and Urban Planning, 80, 95-110.

<https://doi.org/10.1016/j.landurbplan.2006.06.008> 
CRESWELL, John (2013). Research design: qualitative, quantitative, and mixed methods approaches. 4a. ed. Thousand Oaks CA: SAGE.

Dextre, Juan Carlos y AvellanedA, Pau (2014). Movilidad en zonas urbanas. Lima: Fondo Editorial de la Pontificia Universidad Católica del Perú.

EEA (2010). Good practice guide on noise exposure and potential health effects. Copenhague: European Environment Agency.

ElVIK, Rune; ERKE, Alena y VAA, Truls (2008). "Trafikksikkerhetshandboken». <http://tsh.toi.no> [Fecha de consulta: 8 de diciembre de 2009].

EUROPEAN COMMISSION (2004). Reclaiming city streets for people, chaos or quality of life. Bruselas: Directorate-General for the Environment.

EwInG, Reid y Clemente, Otto (2013). Measuring urban design. Washington DC: Island Press.

FORRESTER, John y SNELL, Carolyn (2007). «Planning inclusive and sustainable urban regeneration: balancing a visitor-based economy with local needs in the city of York». Journal of Urban Regeneration and Renewal, 1 (1), 69-82.

FruIN, John (1971). Pedestrian: planning and design. Nueva York: Metropolitan Association of Urban Designers and Environmental Planners, Inc.

Garcia Ramon, Maria Dolors; Ortiz, Anna y Prats, Maria (2014). Espacios públicos, género y diversidad: geografías para unas ciudades inclusivas. Barcelona: Icaria Editorial.

GeHL, Jan (2014). Ciudades para la gente. Primera edición en español. Buenos Aires: Ediciones Infinito.

GeHL, Jan y Svarre, Birgitte (2013). How to study public life. Washington DC: Island Press.

Hammond, Victoria y Musselwhite, Charles (2012). «The Attitudes, Perceptions and Concerns of Pedestrians and Vulnerable Road Users to Shared Space: A Case Study from the UK». Journal of Urban Design, 18 (1), 78-97.

Herce, Manuel (2009). Sobre la movilidad en la ciudad. Propuestas para recuperar un derecho ciudadano. Barcelona: Editorial Reverté.

Hernández-SAmpieri, Roberto; Fernández, Carlos y Baptista, Pilar (2014). Metodología de la investigación. México DF: Mc Graw Hill Education.

Holloway, Catherine; SuZuKI, Tatsuto; UCHIYAMA, Hironobu y Tyler, Nick (2010). Application of the Capability Model to Assess Crossfall Gradient Requirements for Attendant Pushing Wheelchairs. TRANSED 2010. Hong Kong.

HuERTA, Jaime (2007). Discapacidad y diseño accesible. Diseño urbano y arquitectónico para personas con discapacidad. Lima: Universidad de Lima.

IRAP (2018). "Target 3 \& 4: What the mean for safer roads globally». Recuperado de <https://www.irap.org/2018/04/targets-3-4-what-they-mean-for-safer-roadsglobally/> [Consulta: enero de 2019].

JOHNSON, Burke y CHRISTENSEN, Larry (2012). Educational research: quantitative, qualitative, and mixed approaches. 4a. ed. Thousand Oaks, CA: SAGE.

KING, Nigel y HORROCKS, Christine (2010). Interviews in qualitative research. Thousand Oaks, CA: SAGE.

LEWIN, Kurt (1951). Field theory in social science. Nueva York: Harper y Brothers.

LID, Inger Marie y Solvang, Per Koren (2016). "(Dis)ability and the experience of accessibility in the urban environment». ALTER, 10 (2), 181-194. <https://doi.org/10.1016/j.alter.2015.11.003>

LiU, Cong (2008). "Cross-sectional data». En: LaVrakas, Paul (ed.). Encyclopedia of Survey Research Methods. Los Angeles, CA: Sage, 2, 170-171. 
Los Angeles County (2011). Model design manual for living streets. EE. UU.: Los Angeles County.

Manchón, Felipe y SAnTAmera, Juan Antonio (1995). Recomendaciones para el proyecto y diseño del viario urbano. Madrid: Ministerio de Obras Públicas, Transportes y Medio Ambiente, Centro de Publicaciones.

Miralles-Guasch, Carme y Cebollada, Àngel (2009). «Movilidad cotidiana y sostenibilidad, una interpretación desde la geografía humana». Boletín de la AGE, 50, 193-216.

Miralles-Guasch, Carme y Marquet SARdÀ, Oriol (2013). «Dinámicas de proximidad en ciudades multifuncionales». CyTET, XLV (177), 503-512.

NACTO (2013). Urban Street Design Guide, 3ra ed. Nueva York: Island Press.

NEUMAN, William (2009). Social research methods: qualitative and quantitative approache. 7a. ed. Upper Saddle River, NJ: Pearson.

OECD (2012). Aging and transport: mobility needs and safety issues. París: Organization for Economic Co-operation and Development.

Ortiz Escalante, Sara y Gutiérrez Valdivia, Blanca (2015). «Planning from below: using feminist participatory methods to increase women's participation in urban planning». Gender \& Development, 23 (1), 113-126.

PALACIOS, Agustina (2008). El modelo social de la discapacidad: orígenes, caracterización y plasmación en la convención internacional sobre los derechos de las personas con discapacidad. Colección Cermies, 36. Madrid: Ediciones CINCA.

PARKIN, John y SMITHIES, Nicola (2012). «Accounting for the needs of blind and visually impaired people in public realm design». Journal of Urban Design, 17 (1), 135-149. <https://doi.org/10.1080/13574809.2012.646139>

Poldma, Tiiu; LabBé, Delphine; Bertin, Sylvain; Grosbois, Éve de; Barile, Maria; Mazurik Kathrina; Desjardins, Michel; Herbane, Hakim y Artis, Gatline (2014). "Understanding people's needs in a commercial public space: about accessibility and lived experience in social settings». ALTER, 8 (3), 206-216. <https://doi.org/10.1016/j.alter.2014.02.007>

PozUeTA, Julio; LAMIQUiz, Francisco y PORTO, Mateus (2009). La ciudad paseable: recomendaciones para la consolidación de los peatones en el planeamiento, el diseño urbano y la arquitectura. Madrid: Ministerio de Fomento.

Prinz, Dieter (1986). Planificación y configuración urbana. Barcelona: Gustavo Gili. PTV (2013). VISSIM 6.0 user manual. Karlsruhe (Alemania).

Radelat, Guido (2003). Principios de ingeniería de tránsito. EE. UU.: Institute of Transportation Engineers.

Rincón, Delio del; Arnal, Justo; Latorre, Antonio y Sans, Antoni (1995). Técnicas de investigación en ciencias sociales. Madrid: Dykinson.

SANZ, Alfonso (2008). Calmar el tráfico: pasos para una nueva cultura de la movilidad urbana. Madrid: Ministerio de Fomento.

- (2005). «El viaje de las palabras». En: Diputació DE BARCElOnA. Mobilitat obligada. SAM. <www1.diba.cat> [Fecha de consulta: 10 de marzo de 2007].

- (1996). Calmar el tráfico. Madrid: Ministerio de Obras Públicas, Transportes y Medio Ambiente.

SORENSEN, Michael y MOSSLEMI, Marjan (2009). Subjective and objective safety. The effect of road safety measures on subjective safety among vulnerable road users. Oslo: Institute of Transport Economics. Norwegian Centre for Transport Research.

SORIA, Juan (2011). Modelo de umbrales para la evaluación ambiental de la movilidad urbana. Tesis. Departamento de Urbanística y Ordenación del Territorio. Granada: Universidad de Granada. 
SuMPTER, David (2006). «The principles of collective animal behaviour». Philosophical Transactions of the Royal Society B Biological Sciences, 361 (1.465), 5-22. <https://doi.org/10.1098/rstb.2005.1733>

Swaine, Bonnie; Labbé, Elphine; Poldma, Tiiu; Barile, Maria; Fichten, Catherine; Havel, Alice; Kehayta, Eva; Mazer, Barbara; Mckinley, Patricia y Rochette, Annie (2014). «Exploring the facilitators and barriers to shopping mall use by persons with disabilities and strategies for improvements: Perspectives from persons with disabilities, rehabilitation professionals and shopkeepers». ALTER, 8, 217-229.

<https://doi.org/10.1016/j.alter.2014.04.003>

Talavera-García, Rubén; Soria-Lara, Julio y Valenzuela-Montes, Luis Miguel (2014). «La calidad peatonal como método para evaluar entornos de movilidad urbana». Documents d'Analisi Geogràfica, 60 (1), 161-187. $<$ https://doi.org/10.5565/rev/dag.55>

TONUCCI, Francesco (2004). La ciudad de los niños. Un nuevo modo de pensar la ciudad. 3ra edición. Buenos Aires: Editorial Lozada, SA.

TRACY, Sarah (2013). Qualitative research methods: collecting evidence, crafting analysis, communicating impact. Malde, MA: Wiley-Blackwell.

TRB (2000). Highway capacity manual 2000. Washington DC: Transportation Research Board. National Research Council.

- (2010). Highway capacity manual 2010. Washington DC: Transportation Research Board. National Research Council.

WeIDMANN, Ulrich (1993). «Transporttechnik der Fussgänger». ETH Zurich, Ivt Report, 90.

WINANCE, Myriam (2016). «Rethinking disability: lessons from the past, questions for the future. Contributions and limits of the social model, the sociology of science and technology, and the ethics of care». ALTER, 10 (2), 99-110. <http://doi.org/10.1016/j.alter.2016.02.005>

ZiMMERMANN, M. (1989). "The nervous system in the context of information theory». En: Schmidt, Robert y Thews, Gerhard (eds.). Human Physiology. Berlín, Heidelberg: Springer, 166-173. 
